\title{
Nanostructured Surfactant-Based Systems for the Removal of Polymers from Wall Paintings: A Small-Angle Neutron Scattering Study
}

\author{
Michele Baglioni, ${ }^{\dagger}$ Debora Berti, ${ }^{\dagger}$ José Teixeira, $^{\star}$ Rodorico Giorgi, ${ }^{\dagger}$ and Piero Baglioni* ${ }^{\dagger}$ \\ ${ }^{\dagger}$ Department of Chemistry and CSGI, University of Florence, via della Lastruccia 3 - Sesto Fiorentino, 50019 Florence, Italy \\ ${ }^{\ddagger}$ Laboratoire Léon Brillouin (CEA/CNRS), CEA Saclay, bât. 563, 91191 Gif-sur-Yvette Cedex, France
}

Supporting Information

\begin{abstract}
Nanostructured soft matter systems represent effective and long-lasting solutions with respect to traditional and often obsolete methodologies for the conservation of works of art. In particular, complex fluids such as micelles and microemulsions are the most performing media for the removal of organic materials from porous supports, like wall paintings or stones. In this Article, we report on the characterization of two systems, EAPC and XYL, which have shown good to optimal performances in the removal of organic polymers from wall paintings. EAPC is a five-components fluid composed of water, sodium dodecylsulfate (SDS), 1-pentanol (PeOH), propylene carbonate (PC), and ethyl acetate (EA), while $\mathrm{XYL}$ is a "classical" o/w microemulsion, where $p$-xylene droplets are stabilized in water by SDS and PeOH. Small-angle neutron scattering (SANS) with contrast variation is used to infer a detailed picture of the structure of these complex fluids, with a particular focus on the partition of the components between the bulk phase and the nanocompartments. We found that, differently from XYL, the EAPC system is neither a microemulsion nor a simple micellar solution, with the cosolvents partitioned between the dispersing phase and the disperse droplets. These different structural features play a key role in defining the cleaning effectiveness and specifically the kinetics of interaction between the nanofluid and the polymeric coating to be removed, which is of paramount importance for the application in the field. Both of these nanofluids are effective in polymer removal, but EAPC is considerably more efficient and versatile. The composition and the structure at the nanoscale determine the capability of removing a broad range of different polymer coatings from porous materials. A representative case study is here described, addressing a particularly challenging conservative issue, which is the removal of a multilayered aged coating that was irreversibly damaging the pictorial layer of the Annunciation Basilica in Nazareth.
\end{abstract}

\section{INTRODUCTION}

Our cultural heritage is constantly threatened by the flow of time. Aging can be slower or faster, depending on the environmental conditions, on the exhibition in public or private spaces, and on the occurrence of unpredictable events. The preservation of cultural heritage has a huge cost in terms of money, time, and labor, but the socio-economic benefits usually pay back the efforts. Inappropriate or wrong interventions, however, may accelerate the degradation processes or even make them irreversible. A typical example is the application of synthetic hydrophobic polymer coatings onto frescoes, with protective and consolidative purposes, which can lead in the medium or long-term to the complete disruption and detachment of the pictorial layer. This practice is nowadays less and less employed, but meanwhile several unique masterpieces have been damaged and are currently in the need of prompt intervention to remove the polymeric films, without alteration of the underneath pictorial layer. Therefore, the search for compatible cleaning tools for the removal of polymeric films has recently acquired a remarkable relevance in the field. According to these considerations, the design of cleaning systems as restoration tools should be tailored to meet specific chemical and physical properties in terms of compatibility and respect of the original materials of the work of art. Moreover, issues of safety for the operators should also be considered.

Over the last 20 years, our group has designed, prepared, and tested several oil-in-water microemulsions, which are considered the most performing media for the removal of organic materials from porous substrates, and, in particular, for the removal of hydrophobic coatings from the surface of wall paintings. These systems have been tested both on laboratory replicas and on real works of art with good or even excellent performances, with respect to traditional cleaning media, that is, organic solvent or solvent mixtures. ${ }^{1-6}$

EAPC (a nanostructured fluid containing ethyl acetate (EA) and propylene carbonate (PC)) and XYL (a microemulsion containing $p$-xylene) are two particularly efficient cleaning systems, which have been successfully used in several case studies to remove different organic coatings from wall

Received: August 28, 2012

Revised: September 30, 2012

Published: October 1, 2012 
paintings. The EAPC system represents one of the most interesting cleaning media that our group has ever developed, because it is effective in the removal of a wide range of different materials. Its versatility is probably due both to the specific solvent blend contained in its formulation and to its nanostructure. In fact, using this system, it is possible to achieve good cleaning results even in complex conservative situations, when poor information is available about the material that has to be removed or when other detergent systems fail. EAPC provides excellent results in the removal of methacrylic/acrylic or vinyl/acrylic copolymers (i.e., Paraloid B72 and Mowilith DM5), but has been successfully tested also in the removal of complex multilayer coatings, as in the case study reported in the last section of this Article.

The EAPC system was thoroughly investigated in laboratory tests, and its interaction mechanism with Paraloid B72 films was described. $^{7-9}$ Similarly, the cleaning performances and the main chemical-physical properties of the XYL system were studied some time ago. ${ }^{2}$ Nevertheless, a complete structural characterization of these systems is still lacking.

In this Article, we report on a SANS study performed on EAPC and XYL, which allowed us to draw some interesting conclusions about the different removal properties of these two nanostructured fluids.

The structural definition of complex colloidal fluids might require considerable efforts, but it is the necessary prerequisite to improve and tune up the efficiency of cleaning media, to overcome the common trial-and-error approach for the removal of a given polymer coating. EAPC and XYL are both amphiphile-based systems, whose components are reported in Tables 1 and 2. Both contain supramolecular aggregates that

\section{Table 1. Components Present in the EAPC Samples} Investigated

\begin{tabular}{lcccccccc} 
sample name & $\mathrm{D}_{2} \mathrm{O}$ & $\mathrm{SDS}$ & $\mathrm{PeOH}$ & $\begin{array}{c}\text { PeOD- } \\
\mathrm{D}_{12}\end{array}$ & $\mathrm{PC}$ & $\begin{array}{c}\text { PC- } \\
\mathrm{D}_{6}\end{array}$ & EA & $\begin{array}{c}\text { EA- } \\
\mathrm{D}_{8}\end{array}$ \\
EAPC-S & $\mathrm{X}$ & $\mathrm{X}$ & & & & & & \\
EAPC-SP & $\mathrm{X}$ & $\mathrm{X}$ & $\mathrm{X}$ & & & & & \\
EAPC-SP $_{\mathrm{D}}$ & $\mathrm{X}$ & $\mathrm{X}$ & & $\mathrm{X}$ & & & & \\
EAPC-SPP & $\mathrm{X}$ & $\mathrm{X}$ & $\mathrm{X}$ & & $\mathrm{X}$ & & & \\
EAPC-SPP $_{\mathrm{D}}$ & $\mathrm{X}$ & $\mathrm{X}$ & $\mathrm{X}$ & & & $\mathrm{X}$ & & \\
EAPC-SP $_{\mathrm{D}} \mathrm{P}$ & $\mathrm{X}$ & $\mathrm{X}$ & & $\mathrm{X}$ & $\mathrm{X}$ & & & \\
EAPC-SPPE & $\mathrm{X}$ & $\mathrm{X}$ & $\mathrm{X}$ & & $\mathrm{X}$ & & $\mathrm{X}$ & \\
EAPC-SPPE $_{\mathrm{D}}$ & $\mathrm{X}$ & $\mathrm{X}$ & $\mathrm{X}$ & & $\mathrm{X}$ & & & $\mathrm{X}$ \\
EAPC- $^{\text {SPP }} \mathrm{E}_{\mathrm{D}}$ & $\mathrm{X}$ & $\mathrm{X}$ & $\mathrm{X}$ & & & $\mathrm{X}$ & & $\mathrm{X}$ \\
\hline
\end{tabular}

Table 2. Components Present in the XYL Samples Investigated

\begin{tabular}{lcccccc}
\multicolumn{1}{c}{ sample } & $\mathrm{D}_{2} \mathrm{O}$ & $\mathrm{SDS}$ & $\mathrm{PeOH}$ & $\mathrm{PeOD}^{-D_{12}}$ & $\mathrm{Xyl}$ & $\mathrm{Xyl}^{-D_{10}}$ \\
XYL-S & $\mathrm{X}$ & $\mathrm{X}$ & & & & \\
$\mathrm{XYL}-S P$ & $\mathrm{X}$ & $\mathrm{X}$ & $\mathrm{X}$ & & & \\
XYL-SP $_{\mathrm{D}}$ & $\mathrm{X}$ & $\mathrm{X}$ & & $\mathrm{X}$ & & \\
XYL-SPX & $\mathrm{X}$ & $\mathrm{X}$ & $\mathrm{X}$ & & $\mathrm{X}$ & \\
XYL-SPX $_{\mathrm{D}}$ & $\mathrm{X}$ & $\mathrm{X}$ & $\mathrm{X}$ & & & $\mathrm{X}$ \\
\hline
\end{tabular}

are mainly formed by sodium dodecylsulphate (SDS) and 1pentanol $(\mathrm{PeOH})$; nevertheless, their structural features at the nanoscale are deeply affected by the presence of the other components (EA and PC in the first case and $p$-xylene in the latter one). While ethyl acetate and propylene carbonate are partially water-soluble (EA $\sim 8.3 \% \mathrm{w} / \mathrm{w} ;$ PC $\sim 20 \% \mathrm{w} / \mathrm{w}$ ), $p$ - xylene is almost immiscible with water. The different water/ aggregate partitioning plays a key role in defining the nanostructure of the aggregates and their effectiveness as cleaning media. A thorough characterization of the structural traits associated with the performance, in terms of polymer removal, is essential to design ever-improved cleaning systems. In particular, we aimed at assessing the role of each component, both from a structural and from a functional point of view.

Small-angle neutron scattering (SANS), the ideal technique to characterize the structure of amphiphile-based complex systems, has been chosen to determine the structural properties of EAPC and XYL. We performed contrast variation experiments exchanging one component at a time with its deuterated counterpart. As reported in Tables 1 and 2, besides the two complete formulations, several other samples were analyzed, which contained only some of the components in a stepwise fashion. These results yielded a detailed picture of the role of each component in the nanostructure of these fluids.

\section{MATERIALS AND METHODS}

2.1. Chemicals. Sodium dodecylsulfate (Sigma-Aldrich, purity $\geq 99 \%$ ), 1-pentanol (Merck, purity $\geq 98.5 \%$ ), ethyl acetate (SigmaAldrich, ACS reagents, purity $\geq 99.5 \%$ ), propylene carbonate (SigmaAldrich, purity 99\%), $p$-xylene (Merck, purity >99.5\%), fully deuterated 1-pentanol (Cambridge Isotope Laboratories, Inc., 98\%), fully deuterated propylene carbonate (Cambridge Isotope Laboratories, Inc., 98\%), fully deuterated ethyl acetate (Sigma-Aldrich, 99.5\%), fully deuterated $p$-xylene (Sigma-Aldrich, 99\%), and $\mathrm{D}_{2} \mathrm{O}$ (EurisoTop, 98\%) were used as received.

2.2. Samples Studied. The composition of the EAPC system (\% w/w) is $\mathrm{H}_{2} \mathrm{O}, 73.3 \%$; SDS, 3.7\%; 1-pentanol, 7\%; ethyl acetate, $8 \%$; propylene carbonate, $8 \%$. The composition of the XYL system (\% w/ w) is $\mathrm{H}_{2} \mathrm{O}, 89.1 \%$; SDS, 3.9\%; 1-pentanol, $6.5 \%$; $p$-xylene, $2.5 \%$. Tables 1 and 2 summarize the composition of each sample studied in this Article. All of the investigated samples were equilibrated at $25{ }^{\circ} \mathrm{C}$.

Each sample of each series of isotopic substitution has been also analyzed with our in-house SAXS (see details below) and by QELS. Hereafter, describing the structural features of an isotopic substitution series of samples, the symbol "*" will be added to the sample name (e.g., EAPC-SP*), regardless of the isotopic composition of each sample. Complete scattering patterns of each sample analyzed are reported in the Supporting Information.

2.3. Small-Angle Neutron Scattering (SANS). Small-angle neutron scattering measurements were performed on the PAXE instrument of the Laboratoire Léon Brillouin, LLB (Saclay, Gif-SurYvette, Paris, France), using two different configurations, to cover the desired $Q$ range $(Q=(4 \pi / \lambda) \cdot \sin (\theta / 2)$, where $\lambda$ is the wavelength of the incident neutron beam and $\theta$ is the scattering angle). In the medium-high- $Q$ configuration, a wavelength of $5 \AA$ was used, with a sample-to-detector distance of $1.8 \mathrm{~m}$. In the low- $Q$ configuration, a wavelength of $11 \AA \AA$ was used, with a sample-to-detector distance of 5 $\mathrm{m}$. The two $Q$ ranges partially overlap, to provide a consistency check for the merging of the two curves. The wavelength resolution (full width at half-maximum), $\Delta \lambda / \lambda$, was less than $10 \%$. The overall $Q$ range investigated is $0.017<Q<0.256 \AA^{-1}$. Scattered neutrons were detected by a two-dimensional $\mathrm{XY}$ position detector with $64 \times 64$ active elements $\left(\mathrm{BF}_{3}\right)$ covering a total area of $4096 \mathrm{~cm}^{2}$. Samples were contained in $1 \mathrm{~mm}$ thick quartz cells and kept at room temperature $\left(25{ }^{\circ} \mathrm{C}\right)$ during the measurements. The scattering intensity was corrected for the empty cell contribution, transmission, and detector efficiency and was normalized to the absolute scale by a direct measurement of the intensity of the incident neutron beam. The integration of the normalized 2D intensity distribution with respect to the azimuthal angle yielded the 1D scattering intensity distribution, $I(Q)$, in $\mathrm{cm}^{-1}$. The reduction of the data was performed using standard routines available at the LLB. The background from the incoherent scattering was determined from the analysis of the Porod plot and 
subtracted to the normalized spectra. Micelles were modeled as charged prolate ellipsoidal particles interacting with each other according to a screened Coulomb potential described by the NARMMSA (nonadditive radius multicomponent mean sphere approximation). Experimental data normalized to absolute scale were fitted using a NIST routine ${ }^{10}$ (NCNR_SANS_package_6.011 available from NIST - National Institute for Standard and Technology, Gaithersburg, MD) modified by us, running on Igor Pro (Version 6.05A).

2.4. Small-Angle X-ray Scattering (SAXS). Small-angle X-ray scattering (SAXS) measurements were performed with a HECUS S3MICRO SWAXS-camera, equipped with a Hecus System3 2D-point collimator (min divergence $0.4 \times 0.9 \mathrm{mrad}^{2}$ ) and two positionsensitive detectors (PSD-50M) consisting of 1024 channels with a width of $54 \mu \mathrm{m}$. During the experiments, the $\mathrm{K} \alpha$ radiation $(\lambda=1.542$ $\AA$ ) emitted by a $\mathrm{Cu}$ anode from the Oxford $50 \mathrm{~W}$ microfocus source with customized FOX-3D single-bounce multilayer point focusing optics (Xenocs, Grenoble) was used, while the $\mathrm{K} \beta$ line was removed by a multilayer filter. The voltage is generated by the GeniX system (Xenocs, Grenoble). The sample-to-detector distance was $26.9 \mathrm{~cm}$. The volume between the sample and the detector was kept under vacuum during the measurements to minimize the scattering from the atmosphere. The camera was calibrated in the small-angle region using silver behenate $(d=58.38 \AA)$. Scattering curves were obtained in the Q-range between 0.003 and $0.6 \AA^{-1}$. The temperature control was set to $25{ }^{\circ} \mathrm{C}$. Samples were contained in $1.5 \mathrm{~mm}$ thick quartz capillary tubes sealed with hot-melting glue. Scattering curves were corrected for the empty capillary contribution considering the relative transmission factors. Desmearing of the SAXS curves was not necessary thanks to the sophisticated focusing system.

\section{MODELING OF THE AGGREGATES AND DATA ANALYSIS}

3.1. Modeling of the Aggregates. All samples were assumed to be composed of monodisperse prolate ellipsoidal supramolecular aggregates, having an effective charge $Z$ and interacting according to a screened Coulomb potential. The adopted model is consistent with the available literature data on $\mathrm{SDS} / \mathrm{PeOH}$ aggregates in the investigated concentration range, which show a preferential growth along one axis. ${ }^{11-15}$ The only exception with respect to this model is represented by the XYL$\mathrm{SPX}^{*}$ system, that is, the xylene-in- $\mathrm{D}_{2} \mathrm{O}$ microemulsion, because it is well-known that most microemulsions systems are usually characterized by polydisperse spherical aggregates. Figure 1 reports a sketch of the main model used for data analysis, that is, a core-shell aggregate, where $a$ is the major semiaxis, $b$ is the minor semiaxis, and $t$ is the shell thickness. Every region of this model is characterized by a scattering length density (SLD), $\rho_{\text {solv }}, \rho_{\text {shell }}$, and $\rho_{\text {core }}$. For axial ratios ( $a$ / b) up to 1.3 , this model is equivalent to that of polydisperse

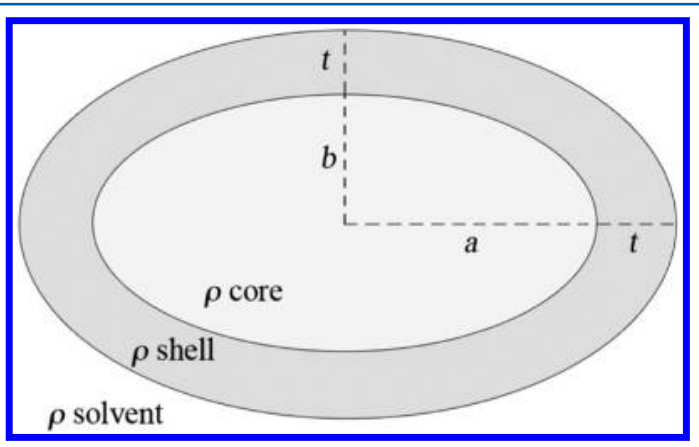

Figure 1. Geometrical description of the model used for the aggregates of EAPC and XYL. They were described as prolate ellipsoidal charged interacting particles. core-shell spheres. ${ }^{15}$ For higher axial ratios (as in the present work, see below), this ambiguity is removed, and the micelles can be considered as actually elongated.

One should be aware that for a multicomponent system, a core-shell model should be considered with due caution: in particular, the shell thickness $t$ might possess only partial physical meaning, giving a coarse estimation of a region of the micelle having different scattering length density with respect to the droplet. Nevertheless, we believe that this more complex fitting model provides, within reasonable uncertainty, a clearer and more detailed picture of the aggregates structure.

In the following paragraphs, the main points of our model will be discussed, highlighting the caveats and the assumptions made. We start from the simplest approach possible, that is, homogeneous micelles, in terms of scattering length density. Further details, as the core-shell model and the other components constituting the dispersed system, are then discussed.

For globular micelles of homogeneous scattering length density, the total scattered intensity $I(Q)\left(\mathrm{cm}^{-1}\right)$ is given by the following equation: ${ }^{16,17}$

$$
I(Q)=N_{\mathrm{p}} V_{\mathrm{p}}^{2} \Delta \rho^{2} P(Q) S(Q)
$$

where $N_{\mathrm{p}}$ is the number density of the scattering objects $\left(\mathrm{cm}^{-3}\right), V_{\mathrm{p}}$ is the volume $\left(\mathrm{cm}^{3}\right), \Delta \rho$ is the contrast (i.e., the difference between the average SLD of the micelle with respect to the SLD of the solvent, $\left.\mathrm{cm}^{-2}\right), P(Q)$ is the form factor function, and $S(Q)$ is the structure factor function.

In the case of asymmetric micelles with a core-shell scattering length profile, $\bar{P}(Q)$ is introduced as the orientationally averaged normalized form factor, and $\bar{S}(Q)$ is the orientationally averaged center-center structure factor among micelles.

The orientation-dependent form factor $F(Q \mu)$, where $\mu$ is the cosine between the direction of the symmetry axis of the ellipsoid and the $Q$ vector, is defined as:

$$
F(Q, \mu)=f \frac{3 j(u)}{u}+(1-f) \frac{3 j(v)}{v}
$$

where $u$ and $v$ are expressed as:

$$
\begin{aligned}
& u=Q\left[\mu^{2} a^{2}+\left(1-\mu^{2}\right) b^{2}\right]^{1 / 2} \\
& v=Q\left[\mu^{2}(a+t)^{2}+\left(1-\mu^{2}\right)(b+t)^{2}\right]^{1 / 2}
\end{aligned}
$$

which define the geometrical shape of the micelles. Considering the simplest case of SDS micelles in $\mathrm{D}_{2} \mathrm{O}, f$ is described by the following equation:

$$
f=\frac{V_{\text {tail }}\left(\rho_{\text {core }}-\rho_{\text {shell }}\right)}{\sum b_{i}-V_{\mathrm{SD} S} \rho_{\mathrm{D}_{2} \mathrm{O}}}
$$

$\bar{P}(Q)$ and $\bar{S}(Q)$ are then evaluated as follows: ${ }^{18}$

$$
\begin{aligned}
& \bar{P}(Q)=\int_{0}^{1} \mathrm{~d} \mu|F(Q, \mu)|^{2} \\
& \bar{S}(Q)=1+\frac{\langle|F(Q, \mu)|\rangle^{2}}{\left\langle|F(Q, \mu)|^{2}\right\rangle}\left[S_{\mathrm{MM}}(Q)-1\right]
\end{aligned}
$$

where $S_{\mathrm{MM}}(Q)$ is the structure factor that accounts for the interactions between the macro-ions, and it is calculated using the MMSA theory (Multicomponent Mean Spherical Approx- 
imation). For an exhaustive treatment of this theory, the reader should refer to the literature in the field. ${ }^{12,16,19-22}$

The calculation of the contrast term, $\Delta \rho$, is very important, especially when performing contrast variation experiments, which require particularly accurate values. For a simple binary system, such as SDS micelles in $\mathrm{D}_{2} \mathrm{O}$, the calculation can be easily handled knowing the value of $V_{\mathrm{SDS}}$, which is the volume of the micellized surfactant contained in the system, and $V^{\mathrm{m}}{ }_{\mathrm{D} 2 \mathrm{O}}$, which is the volume of the $\mathrm{D}_{2} \mathrm{O}$ hydrating the polar heads of the surfactant (i.e., the $\mathrm{D}_{2} \mathrm{O}$ molecules bound to the micelles). In this case, if we assume that the concentration of free surfactant is negligible with respect to that of the associated surfactant (which is a reasonable assumption in the present case where SDS concentration $\gg \mathrm{CMC}$ ), the only uncertainty concerns the hydration number $N_{s}$, that is, the number of $\mathrm{D}_{2} \mathrm{O}$ molecules associated with a micelle for each molecule of SDS. Once this parameter is known or guessed, $\Delta \rho$ can be calculated.

When a cosurfactant $(\mathrm{PeOH})$, other cosolvents, or oils are present (i.e., EA and PC in EAPC or xylene in XYL), for an exact evaluation of the contrast term we have to hypothesize both the hydration number $N_{\mathrm{s}}$ and the partition coefficients $P_{i}$ between the bulk and the micellar phase for the other components. The contrast term calculation for the EAPCSPPE is more complex, and $P_{\mathrm{PeOH}}, P_{\mathrm{PC}}$, and $P_{\mathrm{EA}}$ which are the partition coefficients for pentanol, propylene carbonate, and ethyl acetate, have to be introduced (e.g., for pentanol, $P_{\mathrm{PeOH}}$ is the ratio between alcohol concentration in the micellar phase and its total concentration, $\left.P_{\mathrm{PeOH}}=[\mathrm{PeOH}]_{\mathrm{MIC}} /[\mathrm{PeOH}]_{\mathrm{TOT}}\right)$.

Considering the core-shell separation in terms of coherent scattering length densities, as in the present case, the evaluation is utterly complicated by the partition between the core and the shell of the micelle for each component, or for different portions of the same components (i.e., for the tail and the head of the SDS molecule).

3.2. Fitting Procedure. SANS data were analyzed according to the following procedure (the steps here reported refer to the ellipsoidal model used for most of samples analyzed; a slightly different procedure has been used to fit the XYL-SPX* samples, as detailed in section S4 of the Supporting Information):

(1) The exact composition of each system in term of mass (g) and volume $\left(\mathrm{cm}^{3}\right)$ is the starting point.

(2) A set of initial values for the partition coefficients $P_{i}$, for the hydration number $N_{s}$, and the fractional micellar charge $\alpha=Z / N_{\mathrm{ag}}$ have been assumed, and then refined in the fitting procedure. $N_{\mathrm{ag}}$ is the micellar aggregation number defined in terms of the number of SDS molecules (the only charged species) per micelle. The $P_{i}$ were set according to polarity and water miscibility of the cosolvents and the data available in the literature. ${ }^{3,4,23}$ The value for $N_{\mathrm{s}}$ was chosen according to the literature data available $e^{15,16}$ for SDS micelles (around 9), while for the increasingly complex systems it can reach $25-30$. It is worth noting that the number of $\mathrm{D}_{2} \mathrm{O}$ molecules is referred only to the SDS molecules forming the micelle, even if other components are present, which can be hydrated. The initial fractional charge, $\alpha$, was assumed to be $\sim 0.25^{11,16}$ for SDS micelles.

(3) Given the set of values of the previous paragraph, the initial value for the volume fraction of the micellar phase, $\Phi=V_{\text {mic }} / V_{\text {TOT }}$, was evaluated.
(4) Internal structure of the micelle: the distribution of each component between the core and the shell of the micelle was hypothesized, using the polarity of each component. For example, propylene carbonate, which is a very polar solvent, was located mainly in the shell, in proximity of the polar groups of SDS, while the water-immiscible xylene was assumed to be fully located in the interior of the micelles, shielded from the water phase (see the NMR findings of Palazzo et al. and Colafemmina et al. $^{4,23}$ ).

(5) The initial guess values of the contrast terms, $\rho_{\text {solv }}, \rho_{\text {shell }}$, and $\rho_{\text {core }}$ have been calculated according to the previous assumptions.

(6) The fitting was performed using the IgorPro routine reported in section 2.3. This routine minimizes $\chi^{2}$, giving as output parameters $a, b, t$, and $Z$ (i.e., the geometrical parameters describing shape and size of the micelle and its charge; see Figure 1).

(7) The parameters mentioned in steps (3) and (5) were adjusted by varying the assumptions in steps (2) and (4) until a good fitting curve, based on $\chi^{2}$ minimization, was obtained.

(8) The volume of the single micelle $V_{\mathrm{P}}$ and the core volume $V_{\mathrm{C}}$ were then calculated. From $V_{\mathrm{C}}$ it was possible to evaluate $N_{\mathrm{ag}}$ for the EAPC-S and XYL-S samples:

$$
N_{\mathrm{ag}}=V_{\mathrm{c}} / V_{\text {tail }}
$$

where $V_{\text {tail }}=345.5 \AA^{3}$ is the volume of the aliphatic tail of SDS $\left(-\mathrm{C}_{12} \mathrm{H}_{25}\right) \cdot{ }^{24,25}$ The number of micelles $N_{\text {mic }}$ is then given by:

$$
N_{\text {mic }}=V_{\text {mic }} / V_{\mathrm{P}}
$$

Finally, the composition of the aggregates, that is, the average number of molecules of each component contained in a micelle, was evaluated.

In contrast variation experiments, we have exchanged one by one the components with the corresponding deuterated species. In this case, the above outlined procedure was slightly modified as follows, after step 5 .

(6b) Assuming that the overall structure of the system does not significantly change upon isotopic substitution (see SAXS measurements reported in section 4.1), the data analysis was performed using as input parameters those of the fully hydrogenated samples, and all of the isotopic series was fitted globally. The output parameters $a, b, t$, and $Z$ were constrained within a small range of values, allowed to account for slight variations of the chemical composition and for possible differences arising from the different contrast profile. In fact, for samples with a very different contrast profile, that is, XYL-SP and XYL-SP $\mathrm{D}_{\mathrm{D}}$ the form factor, see eq 1 , can assume different functional forms, in view of the different internal structure of the aggregates, in terms of scattering length distribution, even if the actual shape and size of the aggregates are identical.

(7b) Once obtained the output parameters, the micellar composition was determined as described in step (8).

\section{RESULTS AND DISCUSSION}

4.1. EAPC. Figure 2 reports the complete series of the hydrogenated EAPC samples, where the sole deuterated component is the bulk aqueous phase. The spectra correspond 


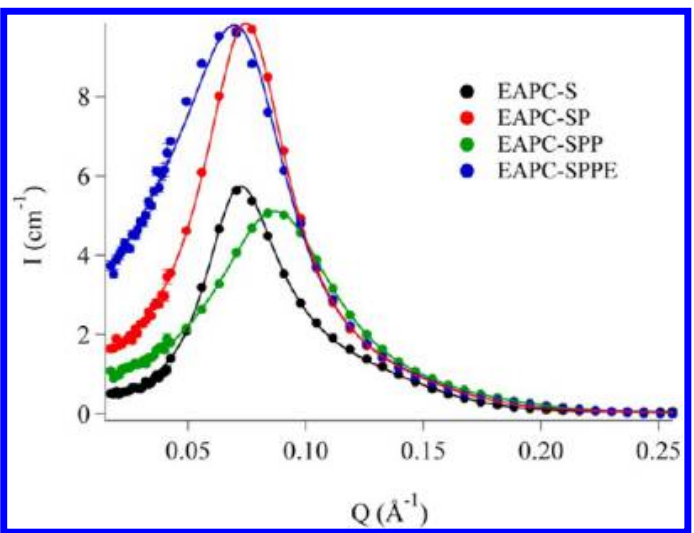

Figure 2. Complete series of the hydrogenated samples for EAPC. Every sample represents a sequential step toward the complete formulation of the nanofluid. The experimental error bars may not be appreciable because they are smaller than the symbols of the experimental data. The fitting curves are represented by continuous lines.

to different chemical compositions, where each ingredient is sequentially added, starting from the binary SDS $/ \mathrm{D}_{2} \mathrm{O}$ sample. From the variation in the absolute scattering intensities and the shift in the peak position, it is clear that each component added affects not only the size, but also the shape and volume fraction of the aggregates.

The variation in forward scattering intensity (i.e., for $Q \rightarrow 0$ ) is due to the combination of varying volume fractions of the scattering objects, $\Phi$, different contrast, and different osmotic compressibility related to the $S(0)$ term.

It is clear that the interpretation of these spectra might be not univocal, as many different factors are involved. However, the SANS technique is unique in this respect, with the $H-D$ isotopic substitution, which allows to detect the localization of the components at a submicellar resolution. It is worth pointing out that the deuteration might change the phase behavior of the systems; ${ }^{26-28}$ however, in the present case, the structural variations due to the isotopic substitution were negligible because both EAPC and XYL systems are in a region of the phase diagram far from any phase transition border.

Figure 3 shows an $\mathrm{H}-\mathrm{D}$ isotopic substitution series of EAPC-SPPE*, that is, the five-component systems differing only for their isotopic composition. In the hypothesis of identical structures, these spectra differ only for the contrast term. In the present case, the structure factor clearly dominates over the form factor; as a result of this feature, the peak position (connected to the order of magnitude of the intermicellar distance) remains constant upon isotopic substitution, and spectral profiles are identical, aside from a multiplicative factor, accounting for the contrast term (for further details, see section S2 in the Supporting Information). To further confirm this observation, SAXS measurements were performed on the very same samples used for SANS. The contrast term in small-angle $\mathrm{X}$-ray scattering is not affected by the isotopic nature of the system. Any observed differences in SAXS should then be ascribed to different structures at the length scales of observation. Figure 4 compares EAPC-SPPE and EAPC$\mathrm{SPPE}_{\mathrm{D}}$ that give identical SAXS patterns, within experimental uncertainty, ruling out any isotopic effect on the microstructure.

A qualitative inspection of the experimental data, flanked by considerations on the chemical properties of the components, is good practice in analyzing SANS results, because it provides

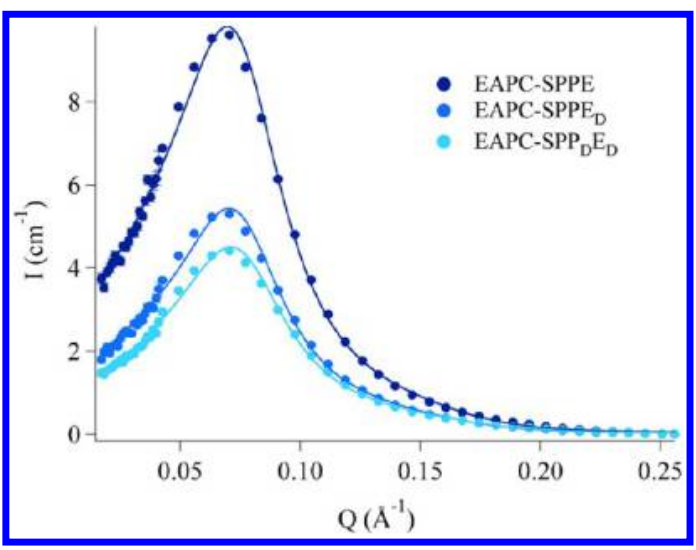

Figure 3. SANS contrast variation measurements on the EAPC complete system (EAPC-SPPE, EAPC-SPPE $\mathrm{D}_{\mathrm{D}}$ EAPC-SPP $\mathrm{D}_{\mathrm{D}}$ ). The fitting curves are represented as continuous lines. The experimental error bars may not be appreciable because they are smaller than the symbols of the experimental data. For a $\log -\log$ version of this graph, see Figure S4 in the Supporting Information.

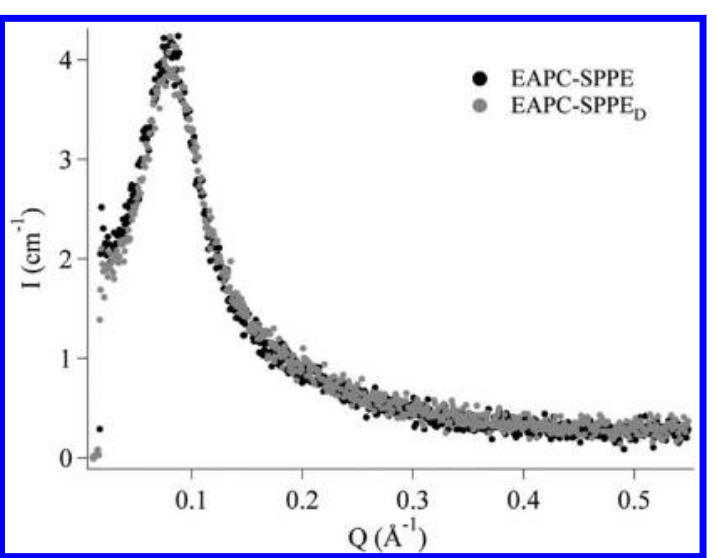

Figure 4. SAXS measurements on samples EAPC-SPPE and EAPC$\mathrm{SPPE}_{\mathrm{D}}$.

useful insights and allows ruling out unphysical results based on $\chi^{2}$ minimization procedures. For instance, it is instructive to combine the observation of the spectra reported in Figure 3 with considerations on the fact that SLD of deuterated PC and EA are very similar (see Table 3 ) and their weight fraction in EAPC is identical. Because of the isotope substitution, the

Table 3. Neutron Coherent Scattering Length Densities of Each Component or Functional Group of EAPC and XYL

$\begin{array}{lc}\text { compound/group } & \mathrm{SLD}\left(10^{-6} \AA^{-2}\right) \\ \mathrm{D}_{2} \mathrm{O} & 6.39 \\ \mathrm{SDS} & 0.39 \\ -\mathrm{C}_{12} \mathrm{H}_{25} & -0.40 \\ -\mathrm{SO}_{4} \mathrm{Na} & 4.46 \\ -\mathrm{SO}^{4-} & 4.50 \\ \mathrm{PeOH} & -0.32 \\ \mathrm{PeOD}-\mathrm{D}_{12} & 6.56 \\ \mathrm{PC} & 1.53 \\ \mathrm{PC}-\mathrm{D}_{6} & 5.97 \\ \mathrm{EA} & 0.52 \\ \text { EA- } \mathrm{D}_{8} & 5.65 \\ \text { xylene } & 0.77 \\ \text { xylene- } & \mathrm{D}_{10}\end{array}$


Table 4. EAPC Fitting Results ${ }^{a}$

\begin{tabular}{|c|c|c|c|c|c|c|c|c|}
\hline sample & $a(\AA)$ & $b(\AA)$ & $t(\AA)$ & $Z\left(1.60 \times 10^{-19} \mathrm{C}\right)$ & $N_{\mathrm{s}}$ & $P_{\mathrm{PeOH}}$ & $P_{\mathrm{PC}}$ & $P_{\mathrm{EA}}$ \\
\hline EAPC-S & $26.1 \pm 0.1$ & $17.2 \pm 0.1$ & $5.2 \pm 0.1$ & $23.7 \pm 0.2$ & 9.2 & & & \\
\hline EAPC-SP & $50 \pm 1.5$ & $11.0 \pm 0.1$ & $11.3 \pm 0.2$ & $13.9 \pm 0.5$ & 33 & 0.9 & & \\
\hline EAPC-SP $_{\mathrm{D}}$ & & & & & & & & \\
\hline EAPC-SPP & $39 \pm 3$ & $9 \pm 2$ & $7.5 \pm 3$ & $13 \pm 1$ & 14.5 & 0.9 & 0.3 & \\
\hline $\begin{array}{l}\text { EAPC-SPP } \\
\text { EAPC-SP } \\
D\end{array}$ & & & & & & & & \\
\hline $\begin{array}{l}\text { EAPC-SPPE } \\
\text { EAPC-SPPE }_{D} \\
\text { EAPC-SPP }_{D} E_{D}\end{array}$ & $64 \pm 4$ & $11.5 \pm 1.1$ & $9.8 \pm 0.5$ & $6.5 \pm 0.3$ & 7 & 0.9 & 0.3 & 0.7 \\
\hline
\end{tabular}

forward intensity decreases in the order: EAPC-SPPE > EAPC$\mathrm{SPPE}_{\mathrm{D}}>\mathrm{EAPC}_{\mathrm{SPP}} \mathrm{E}_{\mathrm{D}}$. A 2-fold intensity drop is due to EA$\mathrm{D}_{8}$ substitution, while the decrease in intensity due to $P C-\mathrm{D}_{6}$ substitution is much smaller: this difference can be seen as an indication of the fact that EA is more abundant than PC in the micellar phase. This is already a first hint about the partition coefficients of the two cosolvents (i.e., $P_{\mathrm{EA}}>P_{\mathrm{PC}}$, which is consistent with the water affinity of the two compounds). Similar considerations have been drawn for all of the curves, to check the consistency of the output values.

Table 4 collects the fitting parameters of the EAPC series, as obtained from the contrast variation experiments. The information gathered made it possible to hypothesize the structure, as illustrated in the following paragraphs.

The first two samples $\left(\mathrm{D}_{2} \mathrm{O} / \mathrm{SDS}\right.$ and $\left.\mathrm{D}_{2} \mathrm{O} / \mathrm{SDS} / \mathrm{PeOH}\right)$ have been thoroughly studied in the literature, but they are nonetheless reported for comparison with the formulations containing PC and EA.

4.1.1. EAPC-S. This system is constituted by SDS micelles in $\mathrm{D}_{2} \mathrm{O}$. The aggregates are slightly elongated, with an axial ratio $a / b$ of 1.5 , too high to account only for a radial polydispersity of globular micelles. ${ }^{15}$ The $b$ axis length $(17.25 \pm 0.04 \AA)$ and the shell thickness $t(5.15 \pm 0.02 \AA)$ match the fully extended hydrocarbon chain and the diameter of the polar head of the SDS molecule. ${ }^{24,25,29}$ The number of $\mathrm{D}_{2} \mathrm{O}$ molecules per SDS is 9.2, in agreement with the literature. ${ }^{16} N_{\mathrm{ag}}$ for this sample was found to be 98, which is slightly higher than what was previously reported for similar systems. ${ }^{11,16}$ We attributed this difference to the fact that SDS was used without further purification, and some long-chain alcohol impurities (common contaminants of SDS) could promote the axial growth of micelles. $^{30}$ The charge of each micelle is 23.7, in good agreement with the 0.23 value reported in the literature for $\alpha$ $\left(Z / N_{\mathrm{ag}}\right){ }^{11,16}$

4.1.2. EAPC-SP*. When $\mathrm{PeOH}$ is added to $S D S / \mathrm{D}_{2} \mathrm{O}$, it distributes between the micellar and the bulk phase, with a partition coefficient $P_{\mathrm{PeOH}}=0.9$. This is a reasonable value, because $\mathrm{PeOH}$ solubility in water is very low $(\sim 2 \% \mathrm{w} / \mathrm{w})$, and nearly the totality of the alcohol is incorporated within the micelles. Candau and Zana have reported that the same alcohol in SDS solutions has a partition coefficient of $0.999^{31}$ at very low, but unspecified, concentrations. In our case, pentanol concentration is higher $([\mathrm{PeOH}]=1.02 \mathrm{M})$, and it is perfectly reasonable that partition is shifted toward the bulk phase. This trend appears consistent with their observations. ${ }^{31}$

The addition of pentanol leads to a pronounced growth of the aggregates along the major axis $(a=50 \pm 1.5 \AA)$, almost 2fold increased with respect to the EAPC-S system. This is a well-known and studied effect, due to intercalation of the 1$\mathrm{PeOH}$ cosurfactant between SDS molecules and consequent lowering of the surfactant film mean curvature. ${ }^{32}$ The interaction between medium length chain alcohols (like pentanol) and SDS has been studied, among others, by Bockstahl et al., Caponetti et al., and Zana et al., 11,13,32 and our results are in line with the published literature. The presence of $\mathrm{PeOH}$ has also the effect of drastically increasing the number of $\mathrm{D}_{2} \mathrm{O}$ molecules per SDS in the micelles, with $N_{\mathrm{s}}$ rising to 33 . The alcohol locates between the surfactant polar heads at the micellar interface that now contains $-\mathrm{OH}$ groups that can interact with $\mathrm{D}_{2} \mathrm{O}$ molecules through hydrogen bonds, resulting in an increased hydration of the aggregates. Also, this finding is consistent with literature data; ${ }^{33}$ however, it is worth noting that, due to the calculations performed by the fitting routine, the value of $N_{s}$ could include uncertainties coming from other fitting parameters. Therefore, this value should be treated as an indication of the amount of $\mathrm{D}_{2} \mathrm{O}$ bound to the micelle rather than the exact number of water molecules per SDS polar head.

4.1.3. EAPC-SPP*. PC is a highly polar and partly watersoluble $(\sim 20 \% \mathrm{w} / \mathrm{w})$ compound; the partition coefficient $P_{\mathrm{PC}}$ was estimated to be 0.3 . Notwithstanding, the solvent fraction that interacts with the micelles is sufficient to produce significant micellar structural changes. One should also consider the possible effects of the dielectric constant of the continuous (solvent) phase, due to the relatively high amount of PC dissolved in $\mathrm{D}_{2} \mathrm{O}$. We have considered possible variations of this parameter in a reasonable range of values on a simulated scattering curve, without appreciable effects. Therefore, the influence of the dielectric constant of the bulk phase on the spectra was considered negligible. Almost the whole volume of PC included in the micelles is located in the shell, where it presumably accommodates between the polar heads of SDS and $\mathrm{PeOH}$ molecules, replacing water molecules $\left(N_{\mathrm{s}}\right.$ is lowered to 14.5). The presence of $\mathrm{PC}$ at the aggregate interface increases the curvature, yielding smaller micelles $(a=39 \pm 2.7$ $\AA$, $b=9 \pm 2 \AA, t=7.5 \pm 3 \AA)$, with an axial ratio $(a+t) /(b+t)$ $=2.8 \pm 1.1$, which indicates that micelles are still elongated along the major axis.

4.1.4. EAPC-SPPE*. With the addition of EA, the complete EAPC formulation is obtained. Like PC, also EA is partly watersoluble $(\sim 8 \% \mathrm{w} / \mathrm{w})$, and thus we expected to find a significant fraction of this solvent in the bulk phase. The fitting results confirmed this hypothesis, because the partition coefficients for the EAPC-SPPE* sample were finally set as $P_{\mathrm{PeOH}}=0.9, P_{\mathrm{PC}}=$ 0.3 , and $P_{\mathrm{EA}}=0.7$. From a structural point of view, the aggregates grow in size both along $a$ and $b(a=64 \pm 4 \AA, b=$ 
$11.5 \pm 1.0 \AA, t=9.8 \pm 0.5 \AA)$. According to the contrast variation fitting results, EA is mainly located in the micelle core.

As was already said, every sample analyzed with SANS was also investigated with SAXS measurements. Here, we describe the results of the SAXS fitting for the EAPC-SPPE sample, whose scattering curve was already reported in Figure 4. The same SANS sample composition was adopted for the SAXS fitting, obtaining a very good fitting. The major semiaxis resulting from the fitting is $a=74.4 \pm 4.8 \AA$, while the minor semiaxis is $b=18.2 \pm 1.4 \AA$. The shell thickness is $t=1.33 \pm$ $0.34 \AA$, which obviously has poor physical significance, given the resolution of the technique. However, if we look at the results of the SANS fitting for the EAPC-SPPE* series, we notice that we obtained almost the same $a+t$ and $b+t$ dimensions from the fitting of the data coming from the two scattering techniques. This is a further confirmation of the reliability of these results. The difference in the value of $a, b$, and $t$ is related to the different contrast profile that the same sample possesses in SANS and SAXS analyses. Figure 5

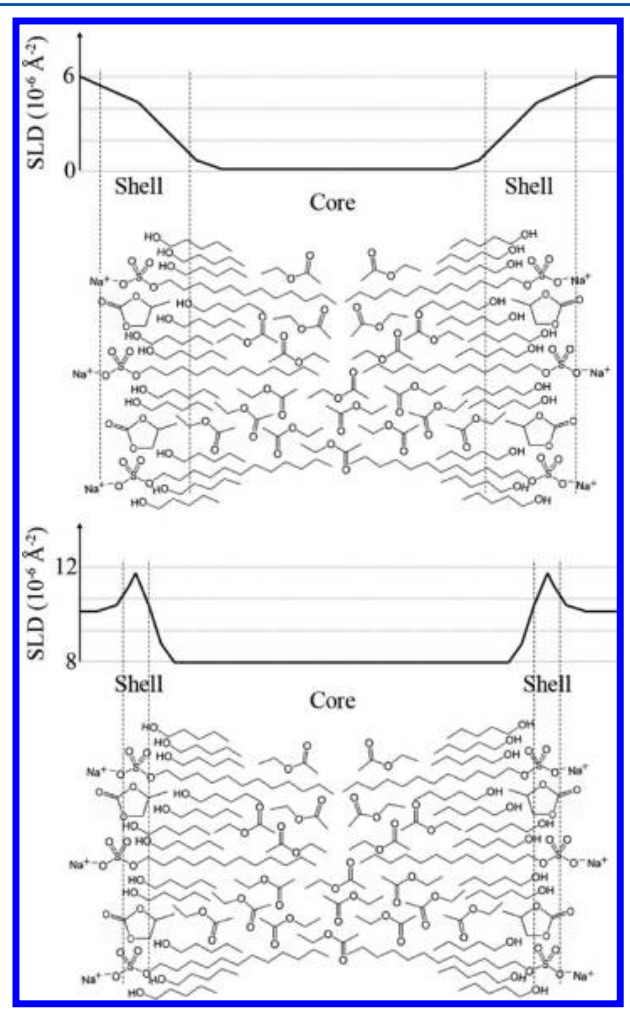

Figure 5. Schematic representation of the scattering profiles of a EAPC-SPPE micellar aggregate longitudinal cross-section for SANS (top) and SAXS (bottom). From the picture, it is noticeable that the contrast profile plays a key role in defining the shell thickness of the same system in the two techniques.

highlights this difference. X-rays are mainly sensitive to SDS polar heads, and in particular to the boundary constituted by sulfur atoms, while neutrons "observe" a smoother and more complex contrast that leads to individuate a wider shell.

4.2. XYL. In Figure 6, the complete series of the fully hydrogenated XYL samples analyzed is reported, while in Table 5 all of the fitting results of XYL system are listed. Comparing SANS spectra reported in Figure 6 with those reported in Figure 2, the difference between XYL and EAPC can be highlighted. The shape and the intensity of the scattering profiles of the XYL-S and the EAPC-S systems are very similar.

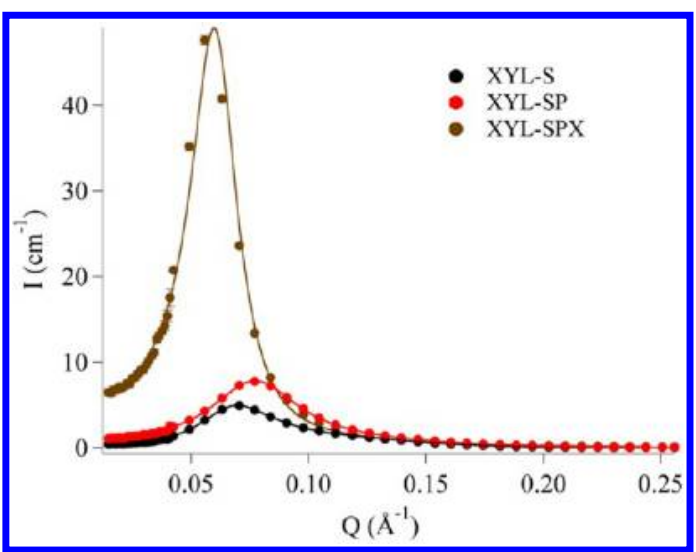

Figure 6. Complete series of hydrogenated samples used for the characterization of the XYL system. Every system represents a building step in the formulation of the nanofluid. Fitting curves are reported together with the experimental points. The experimental error bars may not be appreciable because they are smaller than the symbols of the experimental data.

The same behavior is observed for the samples that contain also $\mathrm{PeOH}$. On the contrary, a difference is appreciable by comparing the EAPC-SPPE and XYL-SPX spectra. The spectrum of the EAPC system comprising all of the components is similar to the EAPC-SP, meaning that, apart from the different contrast profiles of the two samples, the shape, size, and structure of aggregates do not change too much when both PC and EA are added to the micellar system that contains only SDS and $\mathrm{PeOH}$ in $\mathrm{D}_{2} \mathrm{O}$. On the other hand, the SANS profile of the XYL-SPX sample indicates that the addition of xylene to the SDS/PeOH micellar system results in deeper modification of the aggregates. This is an important difference and reflects the different nature of the two systems. As for the EAPC system, it is worth analyzing separately each one of the three steps of the formulation.

4.2.1. XYL-S. This system has a composition similar to that of the EAPC-S system. The fitting of the SANS curve gave results in line with those obtained for EAPC-S, and this is a good confirmation of the reliability of these findings. Also, in this case the aggregates are slightly elongated, their axial ratio is about 1.5 , and their aggregation number is 99 . The same considerations made about the EAPC-S system are valid for XYL-S.

4.2.2. $X Y L-S P *$. When $\mathrm{PeOH}$ is added to the XYL-S micellar solution, we observe the same behavior that is described for the EAPC-SP* system. PeOH partitions itself between the micellar phase and the bulk phase, and the same coefficient, $P_{\mathrm{PeOH}}=0.9$, was found. Also, in this case, the same remarks reported for the EAPC-SP* system apply to this sample. Interestingly enough, $\mathrm{XYL}-\mathrm{SP}^{*}$ contains less $\mathrm{PeOH}$ with respect to EAPC-SP*, and this difference results in a less pronounced axial growth of the aggregates. In fact, while both $b$ and $t$ have almost identical values for XYL-SP* and for EAPC-SP*, micelles contained in the former have a shorter major axis than the ones contained in the latter system. This finding is in good agreement with the results reported in the published literature. ${ }^{11,13}$

4.2.3. $X Y L-S P X^{*}$. Xylene is completely immiscible with water; thus $P_{x y l}$ was set equal to 1 because all of the xylene added to the system should be contained in the micelles. The fitting results confirmed this assumption (Figure 7). While EAPC-SPPE* mixed micelles are significantly elongated, the aggregates of the XYL-SPX microemulsion change from 
Table 5. XYL Fitting Results ${ }^{a}$

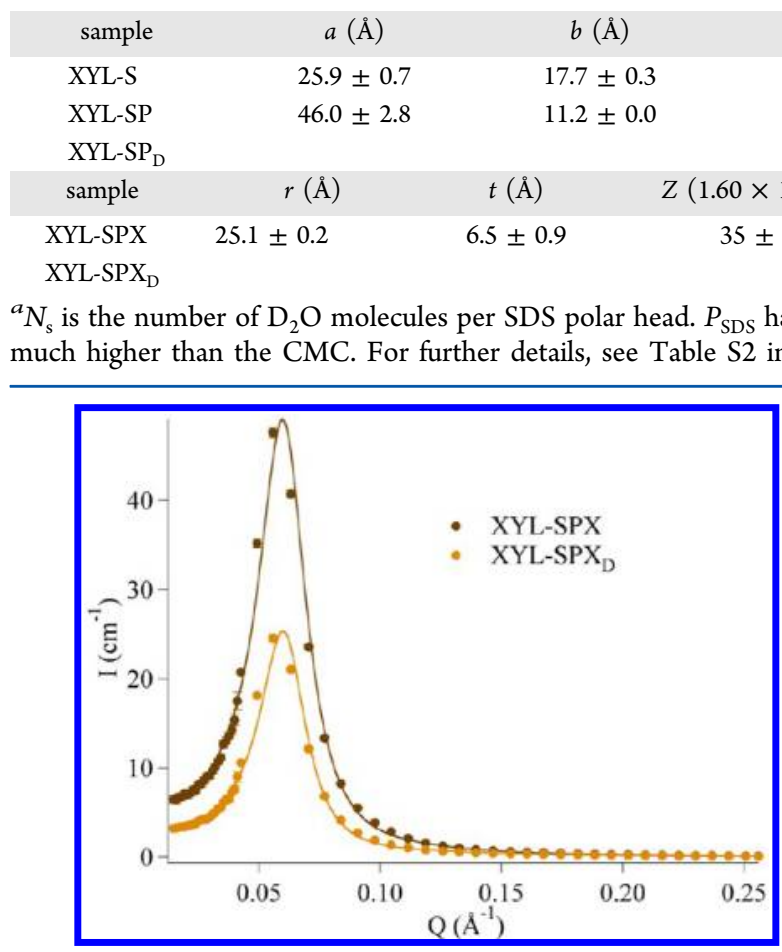

Figure 7. SANS contrast variation measurements on the $\mathrm{XYL}$ complete system (XYL-SPX, EAPC-SPX $\mathrm{D}_{\mathrm{D}}$ ). Fitting curves are reported together with the experimental points. The experimental error bars may not be appreciable because they are smaller than the symbols of the experimental data. For a $\log -\log$ version of this graph, see Figure S7 in the Supporting Information.

ellipsoids to spheres after the addition of xylene. This is a wellknown effect, and it justifies the use of a core-shell spherical fitting model in the analysis of these experimental data. A polydispersity of 0.34 was found for the core radius of the XYLSPX* aggregates. Further details about the fitting of these samples are reported in section S4 of the Supporting Information.

According to these results, the XYL system can be defined as a classical oil-in-water microemulsion constituted by a surfactant (SDS), a cosurfactant $(\mathrm{PeOH})$, and a nonpenetrating oil (xylene), ${ }^{34}$ while the EAPC system is peculiar and cannot be easily classified as a classical microemulsion.

\section{FROM NANOSTRUCTURE TO CLEANING EFFECTIVENESS}

This Article can be framed in the more general context of the development of innovative cleaning media for conservation purposes. In particular, the structural characterization of these systems has be combined with the study of their interaction with the materials to be removed, to unveil the driving mechanisms that lay behind different applicative performances. $^{7-9}$ XYL and EAPC have been tested in the removal of unaged Paraloid B72 from laboratory model samples. According to laboratory tests, $p$-xylene, ethyl acetate, and propylene carbonate are equally good solvents for this polymer, while 1-pentanol only produces some swelling. Therefore, because the surfactant is the same, possible differences in the effectiveness of the two systems must be mainly due to the systems nanostructure or to different amounts of solvents included in the formulation or to a combination of the above factors. As a matter of fact, both systems are able to remove Paraloid from painted surfaces, but a significant difference in the kinetics was observed for the removal process. ${ }^{9}$ Even if EAPC contains almost a 2-fold amount of organic solvents with respect to $\mathrm{XYL}$, this difference has been mainly attributed to a different structure for the two cleaning media, because laboratory tests with EAPC formulations containing reduced quantities of solvents still worked faster than XYL. The SANS investigation reported in this Article further confirms this hypothesis. Indeed, the presence of free solvents dissolved in the bulk aqueous phase of EAPC probably plays a key role in the faster kinetics of this system with respect to XYL. In this latter microemulsion, $p$-xylene and $\mathrm{PeOH}$ are almost completely confined in the dispersed phase and thus less readily available for the interaction with the polymer coating. In other words, the nanostructure influences solvent diffusion coefficient, thus affecting the rate of solvent migration from the nanofluid to the polymer.

\section{CASE STUDY: THE REMOVAL OF HYDROPHOBIC POLYMER COATINGS FROM WALL PAINTINGS IN NAZARETH}

This case study represented a particularly challenging conservative issue. The paintings of the Annunciation Basilica in Nazareth (Israel) were treated in the 1970s with a series of layers of different polymeric materials commercially available at that time. These paintings, dated from fifth century $\mathrm{AD}$, are one of the most ancient representations of the Garden of Eden and are located close to the grotto venerated as the house of Mother Mary. Very little information about the exact chemical nature of the coating was available, and, moreover, during these decades, the polymers visibly degraded, and were discolored, including dust and soil particles, with the final result of making the painting completely unreadable. Moreover, the solubility of the coating in organic solvents was altered due to a combination of chain scissoring and cross-polymerization reactions that usually increase the average molecular weight of the polymer. ${ }^{35}$ Thus, the removal of the altered polymeric materials from the porous supports was really intractable with traditional solvents. Some preliminary tests were carried out with neat organic solvents and with some nanostructured fluids. The XYL microemulsion was also tested, but in this case it did not provide any appreciable results. On the contrary, the more versatile EAPC system gave good results, confirming its excellent cleaning capability.

Thus, polymer removal was carried out loading cellulose pulp poultices with the EAPC systems and letting them act on the painted surface for up to $2 \mathrm{~h}$ (Figure 8 ). At the end of the process, the polymer coating was sensibly swollen and partly removed. Its complete removal then was simply realized by a gentle mechanical action using a cotton swab. In this case study, 


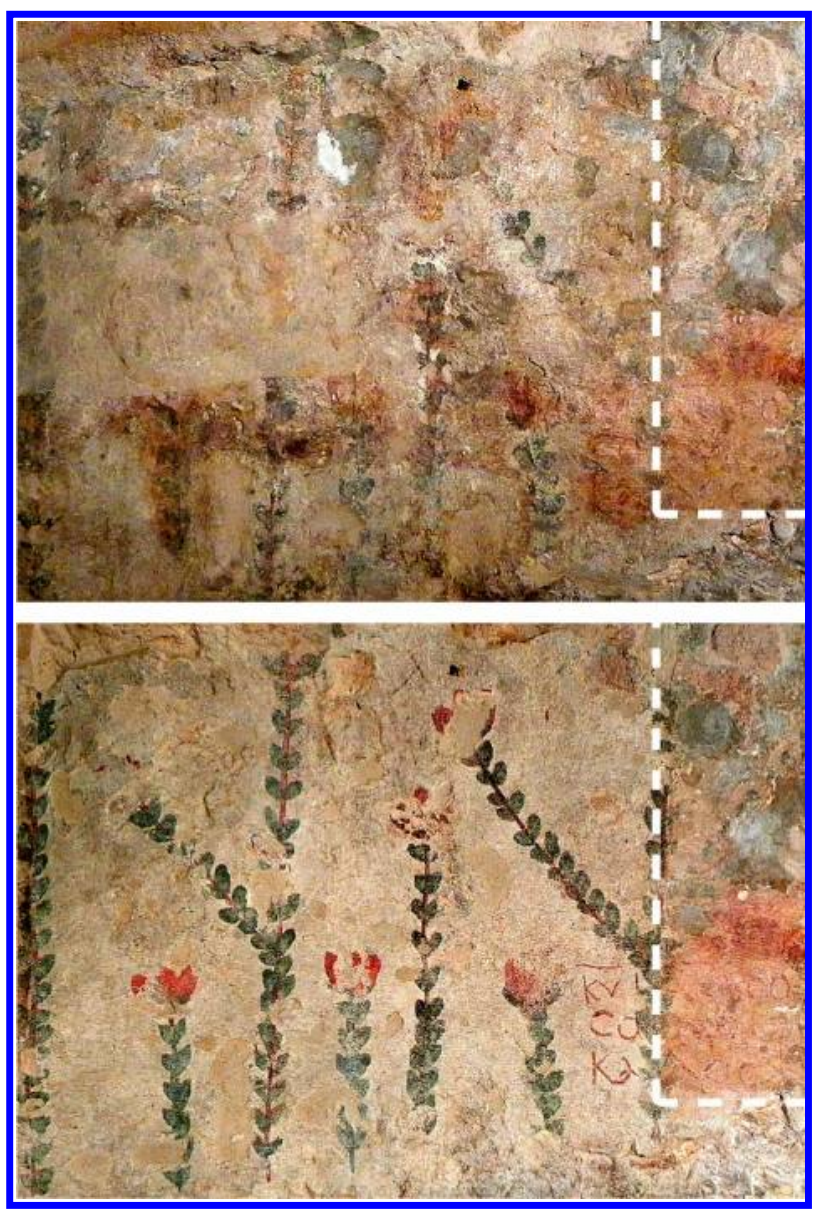

Figure 8. Application of the EAPC system on wall paintings from the Annunciation Basilica in Nazareth (Israel). Top: Before restoration. Bottom: After restoration. In the dashed box, an area is highlighted where the polymer coating has been left untreated as a reference for the evaluation of the cleaning result.

the original colors of the painting were very well conserved, so that, after polymer removal, the readability (and with it, the important cultural meaning, i.e., some ancient inscriptions) of the work of art was completely recovered.

\section{CONCLUSIONS}

In this work, we studied and compared the structure, as deduced from contrast variation small-angle neutron scattering, of two cleaning systems (EAPC and XYL) used for the removal of organic polymers from wall paintings. The analysis of the whole set of data made it possible to get a deep knowledge about the size, shape, and composition of the aggregates present in the two systems.

The EAPC system is constituted by the propylene carbonate and ethyl acetate partly water-soluble solvents; thus only $30 \%$ of PC and $70 \%$ of EA present in the formulation are included in the dispersed phase. The remaining molar fraction is located in the water phase. The addition of these two solvents to SDS/ $\mathrm{PeOH}$ micelles modifies the micelle shape and size, and, in particular, it was found that PC is located among the SDS polar headgroups, while EA intercalates the aliphatic tails of the surfactant. The complex supramolecular aggregates of the EAPC system do not allow an easy definition of the nature of the system itself. It is neither a classical microemulsion (where the oil is considered to be confined in the droplet core), nor a simple micellar solution. These aggregates can be considered as "swollen-micelles", keeping in mind that such a definition cannot be strictly rigorous and that this system represents a "border-line" case.

The XYL system, on the other hand, fits the definition of a classical oil-in-water microemulsion, according to its structure, in which xylene represents the oil phase. This solvent is practically insoluble in water, and this accounts for the fact that it is completely contained in the micellar phase. The aggregates are constituted by a well-defined oil pool separated from the continuous water phase by the surfactant/cosurfactant layer.

Finally, it is important to point out that the main contribution that this work offers with respect to previous studies in the field of nanostructured fluids for the cleaning of artworks is related to the microscopic description and the mechanism of interaction of the two cleaning systems. Until now, we had developed and applied nanostructured systems for polymer removal, and we demonstrated that these are among the most effective media to deal with this conservative issue. The complete structural characterization of two of the most powerful innovative cleaning tools available in conservation of cultural heritage reported in the present study opened new perspectives for future works in view of the correlation between nanostructure and cleaning effectiveness.

\section{ASSOCIATED CONTENT}

\section{S Supporting Information}

Scattering data, contrast variation analysis, fitting results, and fitting procedure. This material is available free of charge via the Internet at http://pubs.acs.org.

\section{AUTHOR INFORMATION}

\section{Corresponding Author}

*E-mail: baglioni@csgi.unifi.it.

\section{Notes}

No kinship exists among the authors.

The authors declare no competing financial interest.

\section{ACKNOWLEDGMENTS}

We wish to thank the conservators Lucia Di Paolo and Tiziana Dell'Omo for the pictures of the paintings and the assistance with the cleaning tests in the Annunciation church in Nazareth, and the "Custodia Terrae Sanctae" for allowing the application of the cleaning systems described in this Article. The access to the LLB neutron facility has been supported by the European Commission under the 7th Framework Program: Integrated Infrastructure Initiative for Neutron Scattering and Muon Spectroscopy: NMI3/FP7 - contract no. 226507. This work has been realized with financial support of "TemArt Programma Operativo Regionale - Regione Toscana, cofinanced by Fondo Europeo di Sviluppo Regionale (POR CreO DESR 2007-2013)", CSGI, and "EU NANOFORART FP7-ENV-NMP-2011 Project ID 282816”.

\section{REFERENCES}

(1) Carretti, E.; Dei, L.; Miliani, C.; Baglioni, P. Oil-in-Water Microemulsions to Solubilize Acrylic Copolymers: Application in Cultural Heritage Conservation. Prog. Colloid Polvm. Sci. 2001, 118, 63-67.

(2) Carretti, E.; Dei, L.; Baglioni, P. Solubilization of Acrylic and Vinyl Polymers in Nanocontainer Solutions. Application of Microemulsions and Micelles to Cultural Heritage Conservation. Langmuir 2003, 19, 7867-7872. 
(3) Grassi, S.; Carretti, E.; Pecorelli, P.; Iacopini, F.; Baglioni, P.; Dei, L. The Conservation of the Vecchietta's Wall Paintings in the Old Sacristy of Santa Maria della Scala in Siena: The Use of Nanotechnological Cleaning Agents. I. Cultural Heritage 2007, 8, 119-125.

(4) Palazzo, G.; Fiorentino, D.; Colafemmina, G.; Ceglie, A.; Carretti, E.; Dei, L.; Baglioni, P. Nanostructured Fluids Based on Propylene Carbonate/Water Mixtures. Langmuir 2005, 21, 6717-6725.

(5) Carretti, E.; Giorgi, R.; Berti, D.; Baglioni, P. Oil-in-Water Nanocontainers as Low Environmental Impact Cleaning Tools for Works of Art: Two Case Studies. Langmuir 2007, 23, 6396-6403.

(6) Carretti, E.; Fratini, E.; Berti, D.; Dei, L.; Baglioni, P. Nanoscience for Art Conservation: Oil-in-Water Microemulsions Embedded in a Polymeric Network for the Cleaning of Works of Art. Angew. Chem. Int. Ed. 2009, 48, 8966-8969.

(7) Giorgi, R.; Baglioni, M.; Berti, D.; Baglioni, P. New Methodologies for the Conservation of Cultural Heritage: Micellar Solutions, Microemulsions, and Hydroxide Nanoparticles. Acc. Chem. Res. 2010, 43, 695-704.

(8) Baglioni, M.; Rengstl, D.; Berti, D.; Bonini, M.; Giorgi, R.; Baglioni, P. Removal of Acrylic Coatings from Works of Art by Means of Nanofluids: Understanding the Mechanism at the Nanoscale. Nanoscale 2010, 2, 1723-1732.

(9) Baglioni, M.; Giorgi, R.; Berti, D.; Baglioni, P. Smart Cleaning of Cultural Heritage: a New Challenge for Soft Nanoscience. Nanoscale 2012, 4, 42-53.

(10) Kline, S. R. Reduction and Analysis of SANS and USANS Data Using Igor Pro. L. Appl. Crustallogr. 2006, 39, 895.

(11) Caponetti, E.; Chillura Martino, D.; Floriano, M. A.; Triolo, R. Localization of n-Alcohols and Structural Effects in Aqueous Solutions of Sodium Dodecyl Sulfate. Lanomuir 1997, 13, 3277-3283.

(12) Liu, Y. C.; Baglioni, P.; Teixeira, J.; Chen, S. H. Structure and Interaction of Lithium Dodecyl-Sulfate Micelles in the Presence of LiSpecific Macrocyclic Cage - A Study By SANS. I. Phys. Chem. 1994, 98, 10208-10215.

(13) Bockstahl, B.; Duplatre, G. Effect of 1-Pentanol on Size and Shape of Sodium Dodecyl Sulfate Micelles as Studied by Positron Annihilation Lifetime Spectroscopy. I. Phys. Chem. B 2001, 105, 1318.

(14) Thimons, K. L.; Bradzil, L. C.; Harrison, D.; Fisch, M. R. Effects of Pentanol Isomers on the Growth of SDS Micelles in $0.5 \mathrm{M} \mathrm{NaCl} I$. Phvs. Chem. B 1997, 101, 11087-11091.

(15) Scaffei, L.; Lanzi, L.; Gambi, C. M.; Giordano, R.; Baglioni, P.; Teixeira, J. Study by Small-Angle Neutron Scattering of Sodium Dodecyl Sulfate Micelles with the Macrocyclic Ligand [2.2.2] Cryptand. I. Phvs. Chem. B 2002, 106, 10771-10776.

(16) Sheu, E. Y.; Chen, S. H. Thermodynamic Analysis of Polydispersity in Ionic Micellar Systems and its Effect on SmallAngle Neutron-Scattering Data Treatment. I. Phvs. Chem. 1988, 92, 4466-4474.

(17) Liu, Y. C.; Ku, C. K.; Lo Nostro, P.; Chen, S. H. Ion Correlations in a Micellar Solution Studied by Small-Angle Neutron and X-Ray Scattering. Phvs. Rev. E 1995, 51, 4598.

(18) Kotlarchyk, M.; Chen, S. H. Analysis of Small Angle Neutron Scattering Spectra from Polydisperse Interacting Colloids. J. Phys. Chem. 1983, 7I9, 2466.

(19) Bendedouch, D.; Chen, S. H.; Koehler, W. C. Determination of Inter-Particle Structure Factors in Ionic Micellar Solutions; Structure and Interparticle Interactions of Bovine Serum Albumin in Solution Studied by Small Angle Neutron Scattering. L. Phys. Chem. 1983, 87, 2621-2628.

(20) Chao, Y. S.; Sheu, E. Y.; Chen, S. H. Experimental Test of a Theory of Dressed Micelles: the Case of the Monovalent Counterion. I. Phvs. Chem. 1985, 89, 4862.

(21) Sheu, E. Y.; Wu, C. F.; Chen, S. H.; Blum, L. Application of a Rescaled Mean Spherical Approximation to Strongly Interacting Ionic Micellar Solutions. Phys. Rev. 1985, 32, 3807.

(22) Chen, S. H. Small Angle Neutron Scattering Studies of the Structure and Interaction in Micellar and Microemulsion Systems. Annu. Rev. Phvs. Chem. 1986, 37, 351.
(23) Colafemmina, G.; Fiorentino, D.; Ceglie, A.; Carretti, E.; Fratini, E.; Dei, L.; Baglioni, P.; Palazzo, G. Structure of SDS Micelles with Propylene Carbonate as Cosolvent: A PGSE-NMR and SAXS Study. L. Phvs. Chem. B 2007, 111, 7184-7193.

(24) Cabane, B. Small Angle Scattering Methods. In Surfactant Solutions New Methods of Investigation; Zana, R., Ed.; Marcel Dekker, Inc.: New York, 1987.

(25) Cabane, B. In Neutron, X-Ray and Light Scattering; Lindner, P., Zemb, T., Eds.; North Holland: Amsterdam, 1991.

(26) Chang, N. J.; Kaler, E. W. The Structure of Sodium DodecylSulfate Micelles in Solutions of $\mathrm{H}_{2} \mathrm{O}$ and $\mathrm{D}_{2} \mathrm{O}$. L Phvs. Chem. 1985, 89, 2996.

(27) Baglioni, P.; Dei, L.; Gambi, C. M. Hydrogen Isotope Substitution in a Water-in-Oil Microemulsion - Quasi-Elastic Light Scattering. I. Phvs. Chem. 1995, 99, 5035.

(28) Cabane, B.; Duplessix, R.; Zemb, T. High-Resolution NeutronScattering on Ionic Surfactant Micelles - SDS in Water. I. Phys. (Paris) 1985, 46, 2161.

(29) Tanford, C. The Hydrophobic Effect: Formation of Micelles and Biological Membranes; Wiley: New York, 1978.

(30) Suzuki, H. Studies of the Effect of 1-Dodecanol on the Micellization of Sodium Dodecyl Sulfate by Gel Filtration. Bull. Chem. Soc. Inn. 1976, 49, 381-383.

(31) Candau, S.; Zana, R. Effect of Alcohols on the Properties of Micellar Systems: III. Elastic and Quasielastic Light Scattering Study. I. Colloid Interface Sci. 1981, 84, 206-219.

(32) Zana, R.; Picot, C.; Duplessix, R. Effect of Alcohol on the Properties of Micellar Systems. V. Small Angle Neutron Scattering Study. J. Colloid Interface Sci. 1983, 93, 43-53.

(33) Baglioni, P.; Kevan, L. Structural Effects of Alcohol Addition to Sodium Dodecyl-Sulfate Micelles Studied by Electron Spin-Echo Modulation of 5-Doxylstearic Acid Spin Probe. I. Phys. Chem. 1987, 91, 1516-1518.

(34) Holmberg, K.; Jonsson, B.; Kronberg, B.; Lindman, B. Surfactants and Polymers in Aqueous Solutions; Wiley: New York, 2004.

(35) Horie, C. V. Materials for Conservation; Butterworth: London, 1987. 